\title{
Signal distortion due to stimulated Raman scattering in optical CDMA transmission systems
}

\begin{abstract}
In this paper, the signal distortion due to stimulated Raman scattering (SRS) in incoherent optical code-division multiple-access (OCDMA) transmission systems is evaluated at different transmission distances. A number of computer simulations have been conducted for this purpose. It is shown that as the total transmit power exceeds Raman threshold level, the SRS will cause severe signal deterioration over long distances even in the absence of the other signal impairment factors.

Keyword: Bit Error Rate (BER) distortion; Nonlinear effects; Nonlinearities; Optical Code Division Multiple Access (OCDMA); Optical transmission; Stimulated Raman scattering (SRS)
\end{abstract}

